\title{
A Unified Numerical Scheme for Calculating Inverse Dynamics of Open/Closed Link Mechanisms
}

\author{
Daigoro Isobe \\ Institute of Engineering Mechanics and Systems, University of Tsukuba \\ 1-1-1 Tennodai Tsukuba-shi \\ Ibaraki 305-8573 \\ JAPAN \\ isobe@kz.tsukuba.ac.jp
}

\begin{abstract}
In this study, a scheme using the Finite Element Method (FEM) for calculating inverse dynamics is proposed and applied to open- and closed-loop link mechanisms. In this scheme, the entire system is subdivided into discrete elements and evaluated as a continuum. A single-link structure of a pin joint and a rigid bar is expressed by using the Shifted Integration (SI) technique. The proposed scheme calculates nodal forces by evaluating equations of motion in a matrix form, and thus information from the entire system can be handled in parallel. The obtained nodal forces are then used to calculate the joint torque in the system. Simple numerical tests on open- and closed-loop link mechanisms are carried out, and it is verified that the scheme can be used as a unified numerical scheme independent of the system configuration.
\end{abstract}

\section{INTRODUCTION}

There is a difficulty in calculating the inverse dynamics for the closed-loop mechanism using conventional methods such as the Newton-Euler method or the Lagrangian method. This is due to the interdependence variables between the constituting links, which become impossible to derive when a chain is closed in the system using the former method. The latter method is also difficult to apply, since the derivation process of an equation considering the binding condition is very complicated. Generally, robotic tasks include motions that generate open and closed loops alternatively, and the dynamic equations of the system (or the numerical algorithm) require an instant revision during the motion. A unified numerical scheme for calculating the inverse dynamics is strongly desired, particularly for those cases of massive, quick-motion robots controlled by force.

Isobe and Nakagawa proposed to apply the Finite Element Method (FEM), a widely used computational tool for analyzing structures, fluids, and so forth, to a control system of connected piezoelectric actuators, and achieved good control not only of the actuator itself but also of the entire system [1]. Isobe et al. implemented the FEM to a calculation scheme of inverse dynamics for hyper-redundant link mechanisms [2]. Using the characteristic of the FEM, which is the capability of expressing the behavior of each discrete element as well as that of the entire continuous system, local information such as nodal forces or displacements can be calculated in parallel. The FEM does not require reimplementation of dynamic equations in the software, and revision can be achieved simply by changing the input data in the case of a physical change in the hardware system.

This study describes a unified numerical scheme for inverse dynamics of two-dimensional link mechanisms. Link mechanisms are modeled using linear Timoshenko beam elements based on the Shifted Integration (SI) technique [3], which was originally used in finite element analyses of framed structures. Nodal forces for obtaining target trajectories are calculated using the FEM, and the joint torque of each link is calculated based on a matrix-formed conversion equation between nodal forces and the joint torque. Some numerical tests are carried out for open- and closed-link mechanisms, to verify the validity of the proposed scheme as a unified numerical scheme independent of the system configuration.

\section{FINITE ELEMENT MODELING OF A LINK MECHANISM}

The SI technique, which is applied in order to model link mechanisms in this study, was originally developed as a finite element scheme for the analysis of framed structures. By considering the equivalence conditions between the strain energy approximations of a linear Timoshenko beam element and a physical model, the rigid-bodies spring model (RBSM), the relationship between the locations of a numerical integration point $\left(s_{1}\right)$ and a plastic hinge $\left(r_{1}\right)$ in the linear Timoshenko beam element $\left(\ddot{A} 1 \hat{\imath} \quad r_{1} ; s_{1} \hat{\imath} 1\right)$ is obtained [3]. Referring to Fig. 1, it is expressed by the following equation:

$\mathrm{s}_{1}=\ddot{\mathrm{A}} \mathrm{r}_{1}$ or $\mathrm{r}_{1}=\ddot{\mathrm{A}} \mathrm{s}_{1}$;

where $s_{1}$ and $r_{1}$ are the positions of the numerical integration point in the finite element and the spring in the RBSM, respectively. Referring to the equation above, the rotational and shear spring placed at the left end $\left(r_{1}=-1\right)$ of an element can be expressed by shifting a numerical integration point in the element to the right end $\left(s_{1}=1\right)$. Various stiffness values of a link joint are then expressed by changing the stiffness of the spring (or the element). Fig. 2 shows the general concept

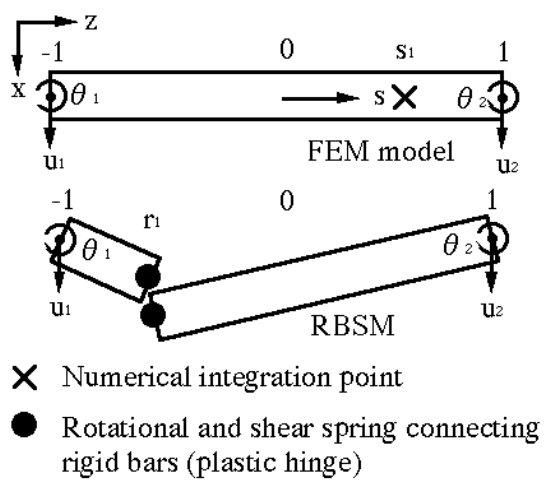

Fig. 1. Linear Timoshenko beam element and its physical equivalent 


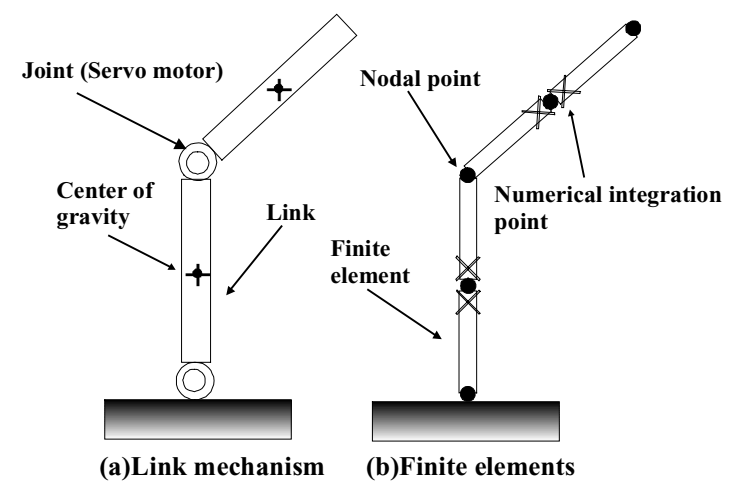

Fig. 2. Modeling of link mechanism by Shifted Integration technique

of modeling by the SI technique. As shown in the figure, a link mechanism formed by a motor and a link member can be modeled by placing a nodal point at the center of gravity, and by two Timoshenko beam elements with numerical integration points shifted to the opposite ends of the link joint. The elemental stiffness matrix is obtained using $s_{1}, r_{1}$ and the normalized stiffness $C_{\text {mot }}$ of the spring, as shown below:

$[K]=C_{\text {mot }}{ }_{V}\left[B\left(s_{1}\right)\right]^{\top}\left[D\left(r_{1}\right)\right]\left[B\left(s_{1}\right)\right] d V$;

Various types of link joints (pin to rigid) can be expressed by varying $C_{\text {mot }}$ between 0 and 1 . The value 0 is used in this study to estimate the validity of the proposed scheme in the pin joint-rigid bar link mechanisms. A lumped mass matrix is also defined using the location of the numerical integration point $s_{1}$. The diagonal components of the elemental mass matrix are:

$[\mathrm{M}]=$

$\left[m_{1} m_{1} m_{1} \frac{m_{1} l^{2}}{12} \frac{m_{1} l^{2}}{12} t_{1} m_{2} m_{2} m_{2} \frac{m_{2} l^{2}}{12} \frac{m_{2} l^{2}}{12} t_{2}\right]$

where

$$
\begin{array}{ll}
m_{1}=\ddot{A} A I\left(1 \ddot{A} s_{1}\right)=2 ; & m_{2}=0 ̈ A I\left(1+s_{1}\right)=2 ; \\
t_{1}=\ddot{o l}_{z} I\left(1 \ddot{A} s_{1}\right)=2 ; & t_{2}=o_{z} I\left(1+s_{1}\right)=2 ;
\end{array}
$$

and $\mathrm{r}, A, l$, and $I_{z}$ are the density of the member, the cross-sectional area, the length of the element and the polar moment of area inertia, respectively. Based on the matrix, the total mass of the element assembles at $r_{1}=1$ when the link joint is placed at $r_{1}=-1$ (thus $s_{1}=1$ ), and vice versa. A nodal point placed between two Timoshenko beam elements thus expresses the center of gravity in a link member (see Fig. 2).

\section{CALCULATION OF JOINT TORQUE IN N-LINK MECHANISM}

Fig. 3 shows the nodal forces (based on global coordinates) acting on the $i$-th link in a two-dimensional closed-loop n-link mechanism, and the configuration of links and nodal points. The joint torque $\tau_{i}$ required on the

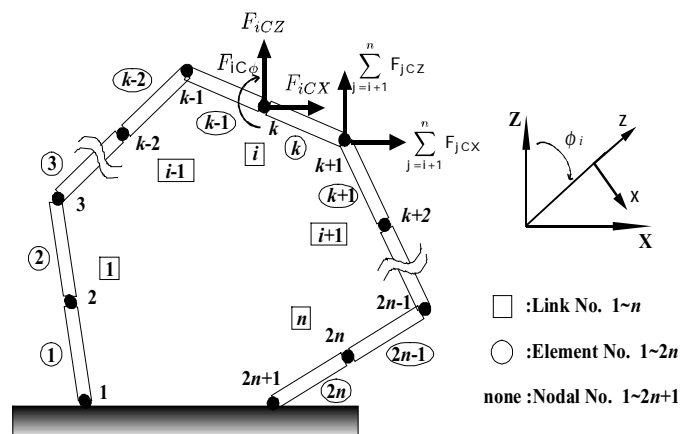

Fig. 3. Nodal forces acting on $i$-th link in closed-loop n-link mechanism

$i$-th link is determined by adding an $i+1$-th joint torque $\tau_{i+1}$ to the sum of inertia moments acting on this link, and is expressed by nodal forces $F_{i C x}$ and $F_{i C \Phi}$ based on elemental (or link) coordinates as follows:

$u_{i}=I_{i C} F_{i C x}+I_{i}\left(X_{j=i+1}^{X^{n}} F_{j C}\right)_{x}+F_{i C u}+u_{+1} ;$

where $l_{i C}$ is the length between the former joint and the center of gravity and $l_{i}$ is the link length. By arranging (5) into global coordinates and in a matrix form, the joint torque vector is expressed as:

$f u^{n} g=\left[L^{n}\right]\left[T^{n}\right] f P^{n} g$

where $\left\{P^{n}\right\}$ is a vector related to nodal force, defined as follows:

$$
\mathrm{fP}^{\mathrm{n} g}=\begin{aligned}
& \mathrm{P}_{1} \\
& \mathrm{P}_{2} \\
& \AA \\
& \AA \\
& \mathrm{P}_{\mathrm{n}}
\end{aligned}
$$

where

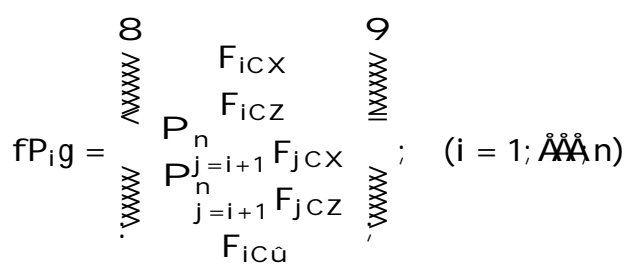

Using the rotational angle $\phi$ between global and elemental (or link) coordinates, the transformation matrix $\left[T^{n}\right]$ is expressed as:

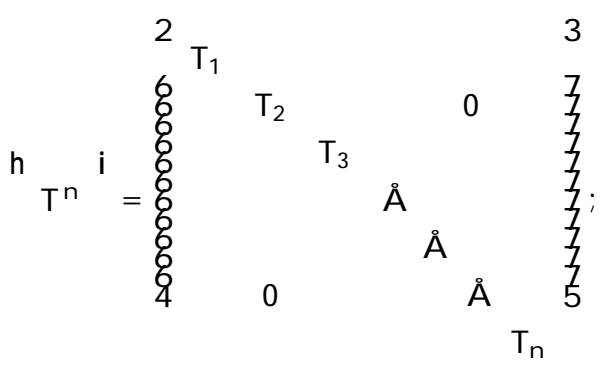


where

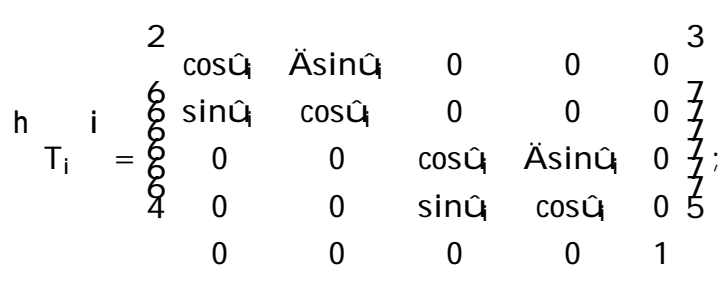

Note that the components are valid for two-dimensional cases. $\left[L^{n}\right]$ is a matrix related to member length, which is expressed as:

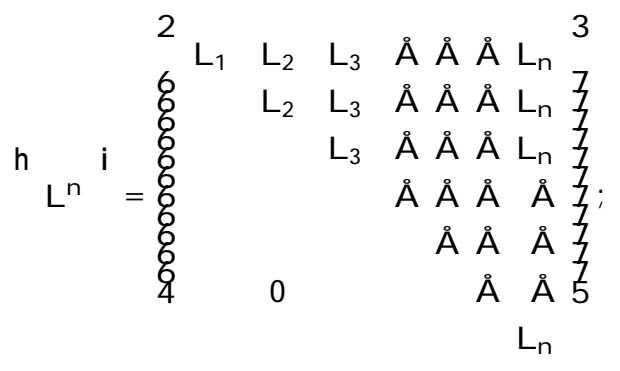

where

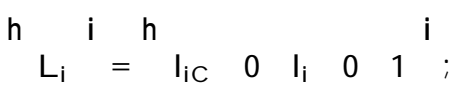

Information on $i+1 \sim n$ link is summed by multiplying the above matrix with vector $\left[T^{n}\right]\left\{P^{n}\right\}$, which is the nodal force vector transformed into elemental coordinates. In cases of closed-loop link mechanisms, the above matrix is divided into multiple parts as shown in the equation below, to fix the configuration of passive joints as well as the torque allocation undertaken by active joints.

$\left[\mathrm{L}^{\mathrm{n}}\right]=\begin{array}{cc}\mathrm{L}^{\mathrm{a}} & 0 \\ 0 & \mathrm{~L}^{\mathrm{b}}\end{array}{ }^{\#} ;$

The suffixes $a$ and $b$ are the number of links $(a+b=n)$ if the mechanism is divided into two parts. This process is the only and the slightest difference of the algorithm between open- and closed-loop link mechanisms. A vector related to incremental nodal forces acting on the $i$-th link is defined using the nodal numbers:

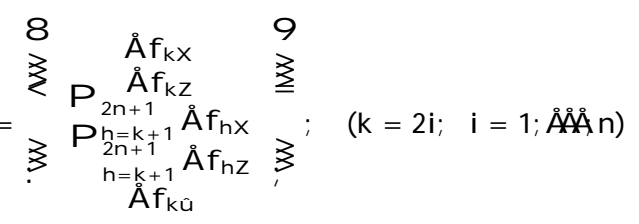

Thus, the vector related to the nodal force acting on the $i$-th link at $\mathrm{t}+\Delta \mathrm{t}$ is successively calculated using the above vector.

$$
f P_{i} g_{t}+\AA t=f P_{i} g_{t}+f \AA p_{k} g ; \quad(k=2 i)
$$

The successive values of the n-link joint torque are then obtained by substituting (15) into (6). Newmark's b method

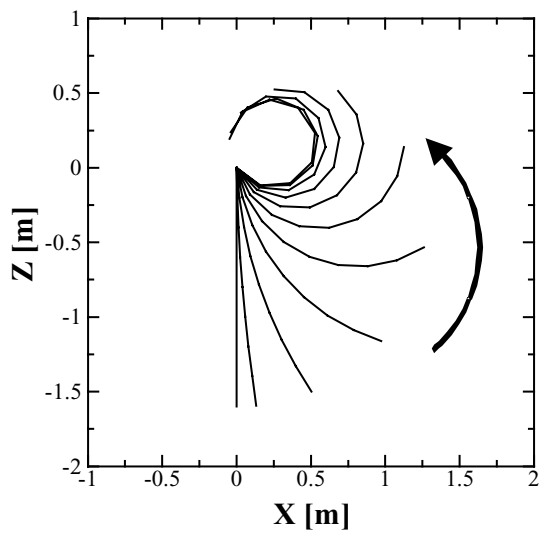

Fig. 4. Target trajectory for open-loop link mechanism

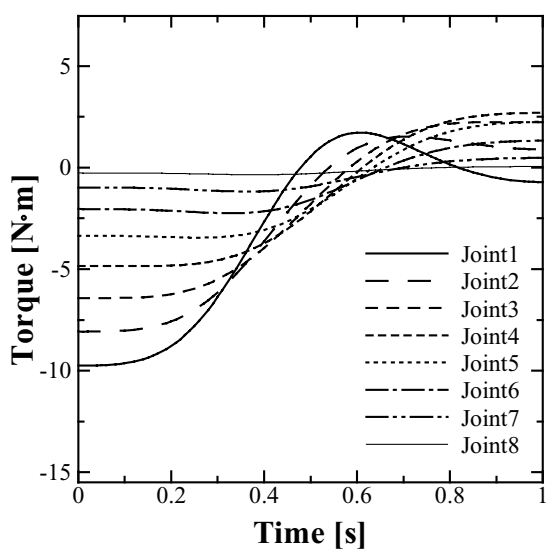

Fig. 5(a). Joint torque curves obtained by Newton-Euler method

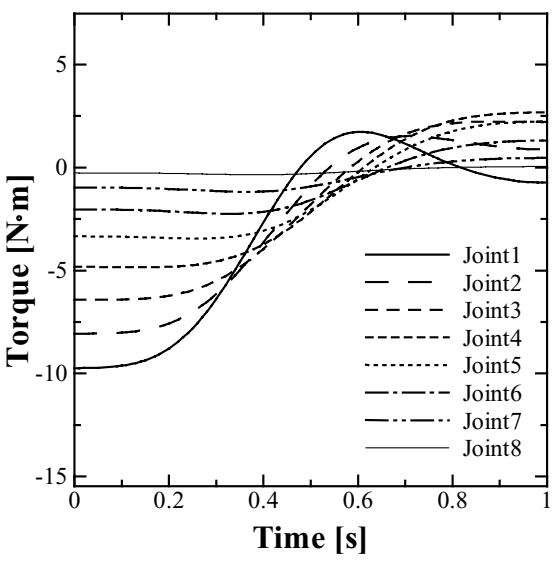

Fig. 5(b). Joint torque curves obtained by FEM

$(d=1 / 2)$ is used as the time integration scheme to solve the incremental kinematic equation.

\section{NUMERICAL EXAMPLES}

First, the proposed scheme using the FEM is applied to the joint torque calculation of an open-loop eight-link mechanism as an example, in order to confirm the accuracy of the calculated torque curves by comparing them with those obtained by the Newton-Euler method. Figs. 5(a) and 5(b) show the torque curves obtained using both schemes 


\section{IECON'01: The 27th Annual Conference of the IEEE Industrial Electronics Society}

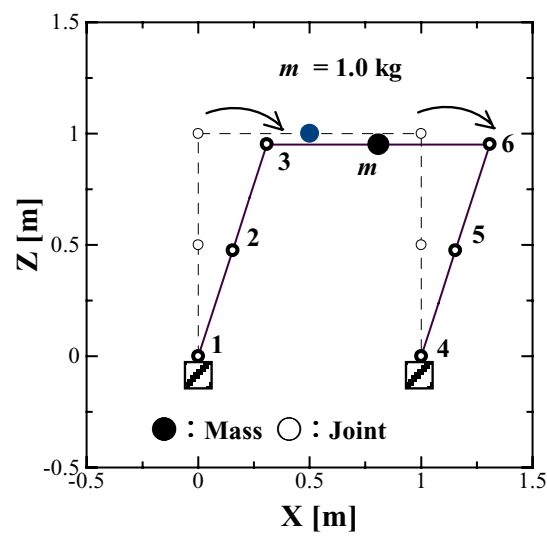

Fig. 6. Target trajectory for closed-loop link mechanism

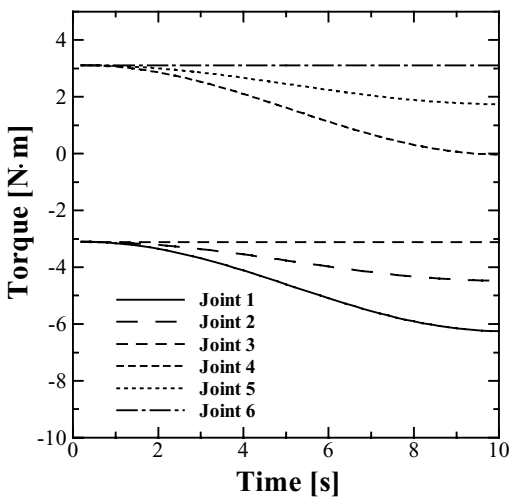

Fig. 7(a). Obtained torque curves (case 1)

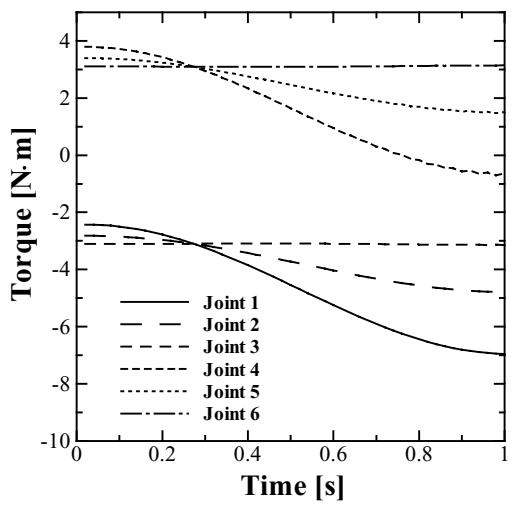

Fig. 7(b). Obtained torque curves (case 2)

when the target trajectory for the 1.0s-motion is given in the eight-link mechanism (length of each link: $20 \mathrm{~cm}$, weight: $107.5 \mathrm{~g}$, center of gravity at midpoint) as shown in Fig. 4. Gravity is assumed to act vertically downward. Although the motion may produce various nonlinear forces such as the Coriolis force, the torque curves obtained by the FEM are in good agreement with those obtained by the Newton-Euler method. Evidently, the proposed scheme is capable of considering every component in the dynamics.

Next, the proposed scheme is applied to the joint torque calculation of a closed-loop six-link mechanism. A simple target trajectory is given in the mechanism (length of each link: $50 \mathrm{~cm}$, weight: $268.75 \mathrm{~g}$, center of gravity at midpoint) as shown in Fig. 6, where a mass of $1.0 \mathrm{~kg}$ is loaded at a passive joint on the lateral beam, driven by six active joints. The same trajectory is given in an operation time of $10.0 \mathrm{~s}$ (case 1) and 1.0s (case 2) to compare the effect of nonlinear forces. The calculated joint torque curves are shown in Figs. 7(a) and 7(b) for the two cases, respectively. The effect of nonlinear forces is negligible in case 1, and thus the initial torque agrees with the required torque at joints 3 and 6 to support the mass $m$ and the link members. The torque values decrease in the minus direction as the mechanism tilts, to prevent the mechanism from collapsing. The torque values at the final stage also agree with the theoretical ones. On the other hand, the effect of nonlinear forces can be observed in case 2, where fluctuations in both initial torque values and amplitude of the torque curves are observed.

\section{CONCLUDING REMARKS}

By deriving the nodal forces in parallel and converting them to the joint torque, the proposed scheme using the FEM can be used to calculate the inverse dynamics of the closed-loop as well as the open-loop link mechanisms without changing the basic numerical algorithm. This unified approach may achieve stability and smoothness in continuous motions of robotic architecture. Construction of the numerical algorithm for three-dimensional cases, and for combined cases with different system configuration is in progress.

\section{ACKNOWLEDGMENT}

The author gratefully acknowledges the contributions of H. Takeuchi, T. Ueda and D. Imaizumi.

\section{REFERENCES}

[1] D. Isobe and H. Nakagawa, "A Parallel Control System for Continuous Architecture Using Finite Element Method", Journal of Intelligent Material Systems and Structures, vol. 9, no. 12, 1999, pp. 1038-1045.

[2] D. Isobe, H. Takeuchi and T. Ueda, "A Numerical Scheme for Calculating Joint Torque of Hyper-Redundant Manipulators -An Approach Using FEM-", Transactions of the Japan Society for Computational Engineering and Science, vol. 2, 2000, pp. 73-78, in Japanese.

[3] Y. Toi, "Shifted Integration Technique in One-Dimensional Plastic Collapse Analysis Using Linear and Cubic Finite Elements", International Journal for Numerical Methods in Engineering, vol. 31, 1991, pp. 1537-1552. 